\title{
Clinical, Economic, and Humanistic Burden Associated With Delayed Diagnosis of Axial Spondyloarthritis: A Systematic Review
}

\author{
Esther Yi (D) - Amit Ahuja - Tanvi Rajput - Aneesh Thomas George • \\ Yujin Park
}

Received: December 5, 2019 / Published online: January 21, 2020

(C) The Author(s) 2020

\begin{abstract}
Introduction: Few studies have evaluated the impact of delayed diagnosis of axial spondyloarthritis (axSpA) on the overall burden of disease. The objective of this review was to evaluate the available literature on the clinical, economic, and humanistic burden of delayed diagnosis in patients with axSpA.

Methods: This systematic literature review was conducted and reported according to the Preferred Reporting Items for Systematic Reviews and Meta-Analyses guidelines. We searched the MEDLINE and Embase databases for Englishlanguage publications of original research articles (up to July 12, 2018) and conference abstracts (January 1, 2014, to July 12, 2018) reporting studies of adult patients with delayed diagnosis of axSpA associated with clinical,
\end{abstract}

Enhanced Digital Features To view enhanced digital features for this article go to: https://doi.org/10.6084/ m9.figshare.11498697.

Electronic Supplementary Material The online version of this article (https://doi.org/10.1007/s40744020-00194-8) contains supplementary material, which is available to authorized users.

E. Yi $(\bowtie) \cdot$ Y. Park

Novartis Pharmaceuticals Corporation, East

Hanover, NJ, USA

e-mail: esther.yi@novartis.com

A. Ahuja - T. Rajput · A. T. George

Novartis Healthcare Pvt Ltd., Hyderabad, India economic, or humanistic burden. Retrieved publications were screened for eligibility by two independent reviewers; discrepancies were resolved by a third independent reviewer. Data were extracted by one reviewer and validated by a second independent reviewer.

Results: A total of 1391 publications were retrieved, of which 21 met the inclusion criteria and were included in the analysis. Of these, 15 reported data on clinical burden, nine on economic burden, and six on humanistic burden, with eight studies reporting a combination of clinical, economic, and/or humanistic burden. Patients with a delayed diagnosis of axSpA generally had higher disease activity, worse physical function, and more structural damage than those who received an earlier diagnosis. Patients with a delayed diagnosis also had a greater likelihood of work disability and higher direct and indirect healthcare costs than those who received an earlier diagnosis. Delayed diagnosis was associated with a greater likelihood for depression, negative psychological impacts, and worse quality of life.

Conclusions: Delayed axSpA diagnosis was associated with more functional impairment, higher healthcare costs, and worse quality of life, highlighting the importance of early recognition of axSpA to reduce extensive burden on patients and society.

Plain Language Summary: Plain language summary available for this article. 
Keywords: Axial spondyloarthritis; Burden of illness; Cost of illness; Delayed diagnosis; Patient-reported outcome measures; Quality of life; Systematic review

\section{Key Summary Points}

This systematic literature review analyzed the available literature on the clinical, economic, and humanistic burden of delayed diagnosis among patients with axial spondyloarthritis (axSpA).

Overall, patients with delayed diagnosis of axSpA had worse clinical outcomes, including higher disease activity, worse physical function, and more structural damage, compared with patients who had an earlier diagnosis

Moreover, patients with a delayed diagnosis had higher healthcare costs and greater likelihood of work disability compared with those with an earlier diagnosis.

Delayed diagnosis was largely associated with worse quality of life, including greater likelihood for depression and negative psychological impact.

Our study emphasizes the value of early recognition of axSpA to enhance clinical outcomes and improve patient and societal burden.

\section{PLAIN LANGUAGE SUMMARY}

Axial spondyloarthritis (axSpA) is a chronic inflammatory disease that primarily affects the spine and can cause chronic back pain and damage to the spinal vertebrae. AxSpA can also cause joint pain, stiffness, fatigue, and reduced physical function, which may lead to considerable physical, economic, and emotional burden. There is often a substantial delay between symptom onset and axSpA diagnosis due to the difficulty of distinguishing back pain associated with axSpA from other forms of back pain, lack of well-established criteria for diagnosis, patient delay in seeking care, and delayed referral of patients to specialists. Delayed diagnosis postpones treatment and disease management, which may result in irreversible structural damage, higher healthcare costs, work disability, and worse health-related quality of life.

Few studies have evaluated the overall impact of delayed diagnosis of axSpA on the burden of disease. This systematic review evaluated the available evidence on the clinical, economic, and humanistic burden associated with delayed diagnosis of axSpA to provide a comprehensive overview of the impact on overall disease burden. Data from 21 relevant studies indicated that patients with delayed diagnosis of axSpA had worse clinical outcomes, including higher disease activity, worse physical function, and more structural damage; higher healthcare costs; greater likelihood of work disability; and worse quality of life, including greater likelihood for depression and negative psychological impact, compared with those who had an earlier diagnosis. This study highlights the importance of early recognition of axSpA to improve outcomes and reduce extensive burden on patients and society.

\section{INTRODUCTION}

Axial spondyloarthritis (axSpA) is a chronic, inflammatory rheumatic disease that primarily affects the axial skeleton [1]. AxSpA encompasses both patients with radiographic sacroiliitis visible on imaging (ankylosing spondylitis [AS]) and those without evidence of radiographic damage in the sacroiliac joints (nonradiographic axSpA). AxSpA is characterized by inflammation of the spinal vertebrae that causes chronic back pain and stiffness and may lead to fusion of vertebral joints. Additionally, axSpA frequently affects the peripheral joints and entheses and is associated with extra-articular manifestations, including uveitis, psoriasis, and inflammatory bowel disease $[1,2]$; if left unmanaged, axSpA may lead to irreversible structural damage and reduced spinal mobility. 
Patients with axSpA can experience considerable physical, economic, and emotional burden due to the pain, fatigue, and impaired physical function resulting from the disease [3-5]. Therefore, early diagnosis and treatment before irreversible changes occur are crucial for managing patients with axSpA.

However, there is often a substantial delay between symptom onset and axSpA diagnosis, with recent reports suggesting an average diagnostic delay of 5-14 years [6-11]. One factor contributing to the delay in diagnosis of axSpA is the difficulty in distinguishing inflammatory back pain (IBP), a key symptom of axSpA that affects the spine and sacroiliac joints [12, 13], from other highly prevalent forms of low back pain in the general population-the overall global prevalence of low back pain is approximately $9 \%$ [14], and up to $80 \%$ of adults will experience low back pain in their lifetime $[15,16]$. Additionally, chronic ( $>3$ months) back pain is a common symptom among several conditions seen in primary care $[17,18]$; prevalence estimates of chronic low back pain vary by country and range from 4 to $24 \%$ [19]. In contrast, the prevalence of AS ranges from 0.01 to $0.54 \%$; among the few studies that have evaluated the prevalence of axSpA in general, prevalence ranges from 0.13 to $1.40 \%$ [20-22]. Thus, IBP due to axSpA may be mistaken for chronic back pain associated with other more common disorders, particularly in patients without clear radiographic sacroiliitis [23].

Another factor contributing to delayed diagnosis is the lack of well-established diagnostic criteria that encompass both AS and nonradiographic axSpA and can be routinely applied in clinical practice. Historically, AS was considered the typical axSpA disease state and was thus the primary focus of classification criteria [21]. The Rome criteria developed in 1961, the New York criteria published in 1966, and the 1984 modified New York criteria require definite sacroiliitis visible on radiographs for classification of AS [24, 25]. However, radiographic sacroiliitis may take years to develop or may not develop at all $[26,27]$, and these criteria therefore do not capture many patients with early AS or nonradiographic axSpA. The Amor criteria (1990) [28] and European
Spondyloarthropathy Study Group criteria (1991) [29] include but do not require radiographic sacroiliitis; although these criteria have been applied for classification of patients with axSpA, they were developed for spondyloarthritis (SpA) in general and emphasize peripheral features of $\mathrm{SpA}$, which may result in misclassification of patients with limited axial involvement.

To better differentiate axSpA from peripheral SpA and allow for the classification of nonradiographic axSpA, the Assessment of SpondyloArthritis International Society (ASAS) developed updated classification criteria for the identification of axSpA in 2009 [30, 31]. Patients must have $\geq 3$ months of back pain, age of onset $<45$ years, and radiographic sacroiliitis on imaging with $\geq 1 \mathrm{SpA}$ feature or HLA-B27 positivity and $\geq 2 \mathrm{SpA}$ features. SpA features include IBP, arthritis, enthesitis, uveitis, dactylitis, psoriasis, Crohn's disease or ulcerative colitis, good response to nonsteroidal antiinflammatory drugs, HLA-B27 positivity, and elevated C-reactive protein (CRP) levels. Overall, the ASAS criteria have good sensitivity and specificity for the classification of axSpA $[31,32]$.

Although greater awareness of axSpA and the development and application of classification criteria have resulted in a decrease in the time to axSpA diagnosis [33-35], the above classification criteria were developed for clinical research and not as diagnostic tools; thus, use of these criteria for diagnosis of axSpA can be limited in routine clinical practice $[1,36,37]$. Criteria such as IBP, good response to nonsteroidal anti-inflammatory drugs, and presence of enthesitis are somewhat subjective in nature [36]. Development of radiographic sacroiliitis may be delayed [26, 27], and interpretation of radiographs of the sacroiliac joints is subject to substantial interreader variability [38, 39]. Current magnetic resonance imaging protocols routinely used in the evaluation of low back pain can yield false-negative results due to low sensitivity for inflammation or false-positive results due to inflammatory changes that can occur in the sacroiliac joints of athletes and patients with degenerative arthritis, trauma, or other conditions [36, 37, 40-42]. Additionally, use of 
these criteria may not be regularly applied in rheumatology settings [43], and discrepancies have been observed between fulfillment of ASAS criteria and rheumatologist diagnosis of axSpA [32].

The majority of patients eventually diagnosed with axSpA first seek care from primary care physicians or nonrheumatology healthcare providers [7, 36, 43, 44]. Prompt referral to rheumatologists can facilitate an earlier diagnosis [36]. However, referral to rheumatology specialists is often delayed due to underrecognition of symptoms of IBP suggestive of axSpA among nonrheumatology healthcare providers $[36,44,45]$. Lack of nearby specialists and long wait times may further inhibit timely referral of patients $[44,45]$. Factors such as patient delay in seeking care, patient reluctance to see a specialist, and insurance restrictions may also contribute to diagnostic delay [44]. Delayed diagnosis of axSpA results in delayed treatment and disease management, which may negatively impact disease prognosis and result in greater economic burden and worse health-related quality of life due to continued disease progression [46]. Few studies have evaluated the impact of delayed diagnosis of axSpA on the overall burden of disease. The objective of this systematic review was to evaluate the available evidence on the clinical, economic, and humanistic burden associated with delayed diagnosis in patients with axSpA to provide a comprehensive overview of the impact of delayed diagnosis on overall disease burden.

\section{METHODS}

\section{Data Sources}

This systematic review was conducted according to the Preferred Reporting Items for Systematic Reviews and Meta-Analyses guidelines [47]. We searched the MEDLINE Literature Analysis and Retrieval System Online (including MEDLINE In-Process) and Excerpta Medica (Embase) databases for original research articles (up to July 12 , 2018) and conference abstracts (January 1, 2014 , to July 12,2018 ) reporting studies of delayed diagnosis of adult patients with axSpA associated with clinical, economic, or humanistic burden. The list of search terms is described in Supplementary Table 1. We also manually searched references cited in Cochrane reviews and/or systematic reviews identified during screening to identify any additional published literature not identified during the database searches. Additionally, abstract archives of the American College of Rheumatology/Association of Rheumatology Health Professionals Annual Meeting and the European League Against Rheumatism Annual European Congress of Rheumatology were searched to identify abstracts not yet indexed in Embase at the time of the search. This article is based on previously conducted studies and does not contain any studies with human participants or animals performed by any of the authors.

\section{Eligibility Criteria and Article Selection}

Publications eligible for inclusion were Englishlanguage, noninterventional, original research studies of adult patients with axSpA that reported association of delayed axSpA diagnosis with clinical (e.g., comorbidities, mortality, disability, and functional status), economic (e.g., direct/indirect costs, resource use), or humanistic (e.g., health-related quality of life or utility measures) burden. The key inclusion and exclusion criteria are described in Table 1.

Abstracts of all records retrieved from the literature search were screened for eligibility by two independent reviewers; any discrepancies were resolved by a third independent reviewer. Citations that did not match the eligibility criteria and duplicates of citations were excluded at the abstract-screening stage. Full-text publications of the included abstracts were retrieved and underwent second-level screening by two independent reviewers, and discrepancies were resolved by a third independent reviewer.

\section{Data Extraction and Quality Assessment}

Data from the final list of included studies were extracted by one reviewer and validated by a second independent reviewer; any discrepancies were resolved by a third independent reviewer. 
Table 1 Details of systematic literature review methodology

Databases

Time frame

Inclusion criteria

Exclusion criteria

Critical appraisal
tools

Data extraction

Electronic databases: MEDLINE, MEDLINE In-Process, and Embase

Conference databases: ACR/ARHP Annual Meeting and EULAR Annual European Congress of Rheumatology archives

Full-text articles: database start to July 12, 2018

Conference abstracts: January 1, 2013, to July 12, 2018

Population: adult patients with axSpA

Outcomes

Clinical: comorbidities, mortality, and disability and functional status (e.g., BASDAI, BASFI, BASMI)

Economic: direct/indirect costs, resource use data, type of cost data and value, and type of resource use and value

Humanistic: HRQOL and utility values and qualitative measures

Study design: original research studies, including observational studies; claims database studies; surveys; and any study reporting cost, resource use, and HRQOL data

Animal/in vitro studies and studies with children or patients with peripheral spondyloarthritis

Non-English-language articles

Interventional studies, including RCTs, nRCTs, or single-arm trials

HTAs, reviews, editorials, case reports, and case series

Downs and Black Quality Index for assessing risk of bias [48]

Total number of patients analyzed, number of patients with outcome, mean, SD, SE, median, range, 95\% CI, and $P$ values, as applicable

$A C R$ American College of Rheumatology, $A R H P$ Association of Rheumatology Health Professionals, axSpA axial spondyloarthritis, BASDAI Bath Ankylosing Spondylitis Disease Activity Index, BASFI Bath Ankylosing Spondylitis Functional Index, BASMI Bath Ankylosing Spondylitis Metrology Index, EULAR European League Against Rheumatism, $H R Q O L$ health-related quality of life, $H T A$ health technology assessment, $n R C T$ nonrandomized controlled trial, $R C T$ randomized controlled trial

For each included study, the study title, country, number of centers, total study population, objective, inclusion/exclusion criteria, followup duration, patient group populations, baseline patient data (age, sex, race, and disease duration), and authors' conclusions were extracted. Outcomes extracted to evaluate clinical burden included pain, Bath Ankylosing Spondylitis Disease Activity Index (BASDAI), Bath Ankylosing Spondylitis Functional Index (BASFI), Bath Ankylosing Spondylitis Metrology Index (BASMI), comorbidities, and disability.
When available, laboratory values (e.g., CRP levels, erythrocyte sedimentation rate [ESR]) and measures of radiographic progression (e.g., Bath Ankylosing Spondylitis Radiology Index, modified Stoke Ankylosing Spondylitis Spine Score [mSASSS]), and spinal mobility (e.g., Schober test, occiput-to-wall distance) were also extracted. For assessment of economic burden, data on type and value of direct and indirect costs, resource utilization (e.g., number and length of hospital stays), and employment or disability were extracted. Outcomes extracted to 
evaluate humanistic burden included Ankylosing Spondylitis Quality of Life questionnaire (ASQOL) and the 36-item Short Form Health Survey.

For dichotomous outcomes, the data extracted were total number of patients analyzed and the number of patients with the outcome. For continuous outcomes, the data were extracted as number of patients, mean, SD, SE, median, range, 95\% CI, and $P$ values, as applicable.

The methodological quality of each study was evaluated using the Downs and Black Quality Index for assessing risk of bias [48]. Briefly, study methodology was assessed using 26 questions evaluating characteristics of study reporting, external validity, and internal validity (bias and confounding) (Supplementary Table 2). The total possible score using this scale was 27 , with higher numbers indicating higher methodological quality/lower risk of bias.

\section{RESULTS}

\section{Study Selection, Characteristics, and Quality Assessment}

The initial search yielded 1391 citations, from which 21 studies were identified for inclusion after screening [6, 35, 46, 49-66] (Fig. 1). Among the included studies, 15 reported clinical burden [6, 35, 46, 49-52, 55, 59-65], nine reported economic burden $[35,46,52,55-58$, $62,66]$, and six reported humanistic burden $[6,53,54,62,65,66]$; eight studies reported data on a combination of clinical, economic, and/or humanistic burden $[6,35,46,52,55,62$, $65,66]$, one of which reported data on all three outcomes [62] (Fig. 2).

Study characteristics are described in Table 2. The 21 included studies were published between 2009 and 2018 and were conducted in 13 countries: Argentina $(n=1)$, Australia

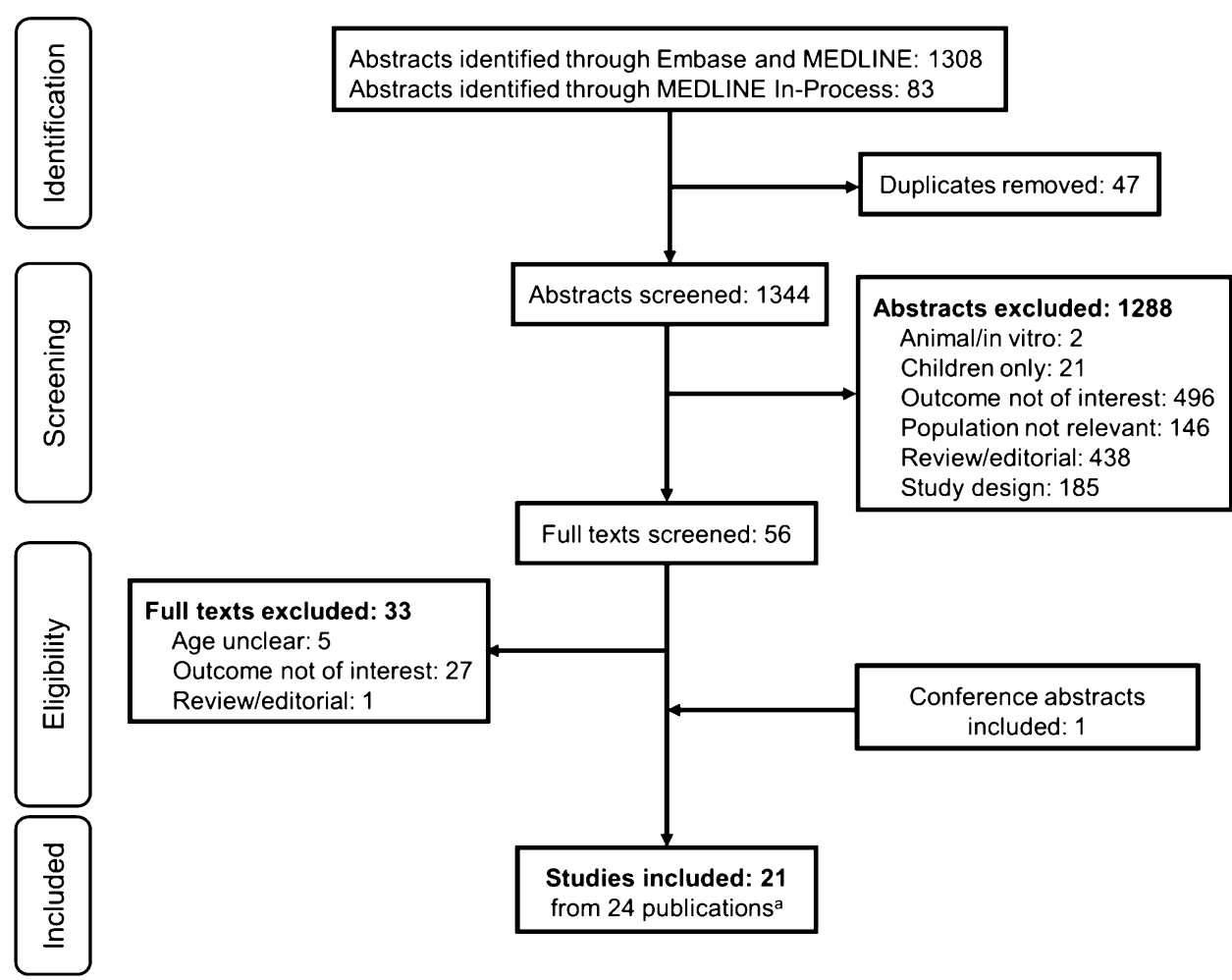

Fig. 1 PRISMA diagram for study selection. Searches were performed on July 12, 2018. PRISMA, Preferred Reporting Items for Systematic Reviews and Meta-Analyses. ${ }^{a}$ Three studies had two publications each 
A Study Outcome Type

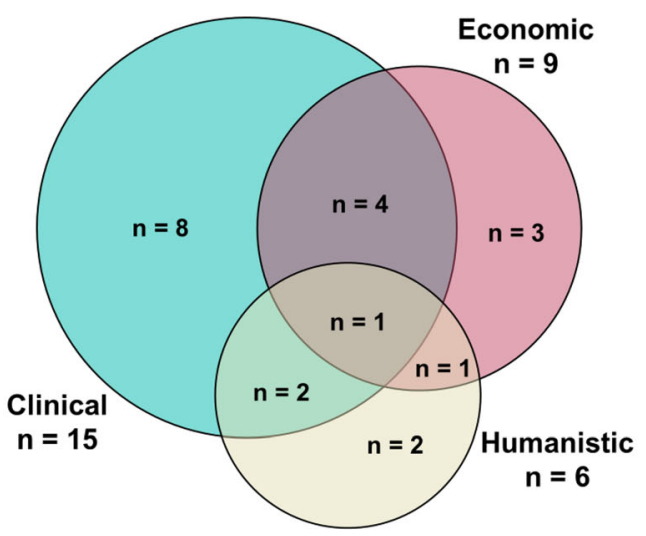

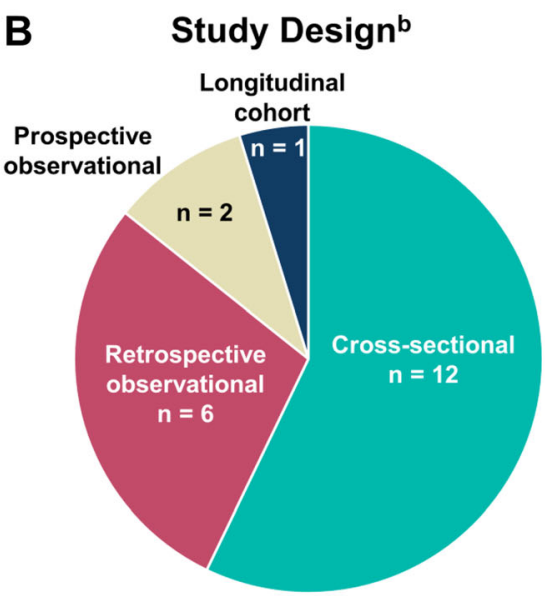

46, 49-51, 56, 58-60, 63, 66; retrospective observational, 52, 54, 55, 57, 61, 64; prospective observational, 62, 65; longitudinal cohort, 53

having a score of $\geq 6$. For the assessment of external validity, scores ranged from 1 to 6 of 10 , with the majority of studies $(n=15)$ having a score of $\leq 4$. For questions assessing internal validity, scores ranged from 0 to 3 of 6 , with 12 studies having a score of 3 .

\section{Clinical Burden of Delayed Diagnosis}

Among the 15 studies that evaluated clinical burden of delayed axSpA diagnosis, the most common measures used to assess disease activity included BASDAI $(n=12)[6,35,46,51,52$, $55,59-63,65]$, BASFI $(n=12)[6,35,46,51,52$, $55,59-63,65]$, and BASMI $(n=8)[6,35,51,52$, $55,61,62,65]$ (Table 3). A significant association was found between longer diagnosis delay and worse BASFI scores in eight of 12 studies $[6,35,46,51,59-62]$ and worse BASMI scores in six of eight studies $[6,35,51,52,61,62]$; however, only four of 12 studies found a significant association between longer delay and worse BASDAI scores [6, 35, 46, 51]. Additionally, one study reported that patients with delayed axSpA diagnosis responded less favorably to treatment based on BASDAI scores and the rate of radiographic progression [46].

Five studies evaluated additional measures of spinal mobility (e.g., Schober test, occiput-to- 


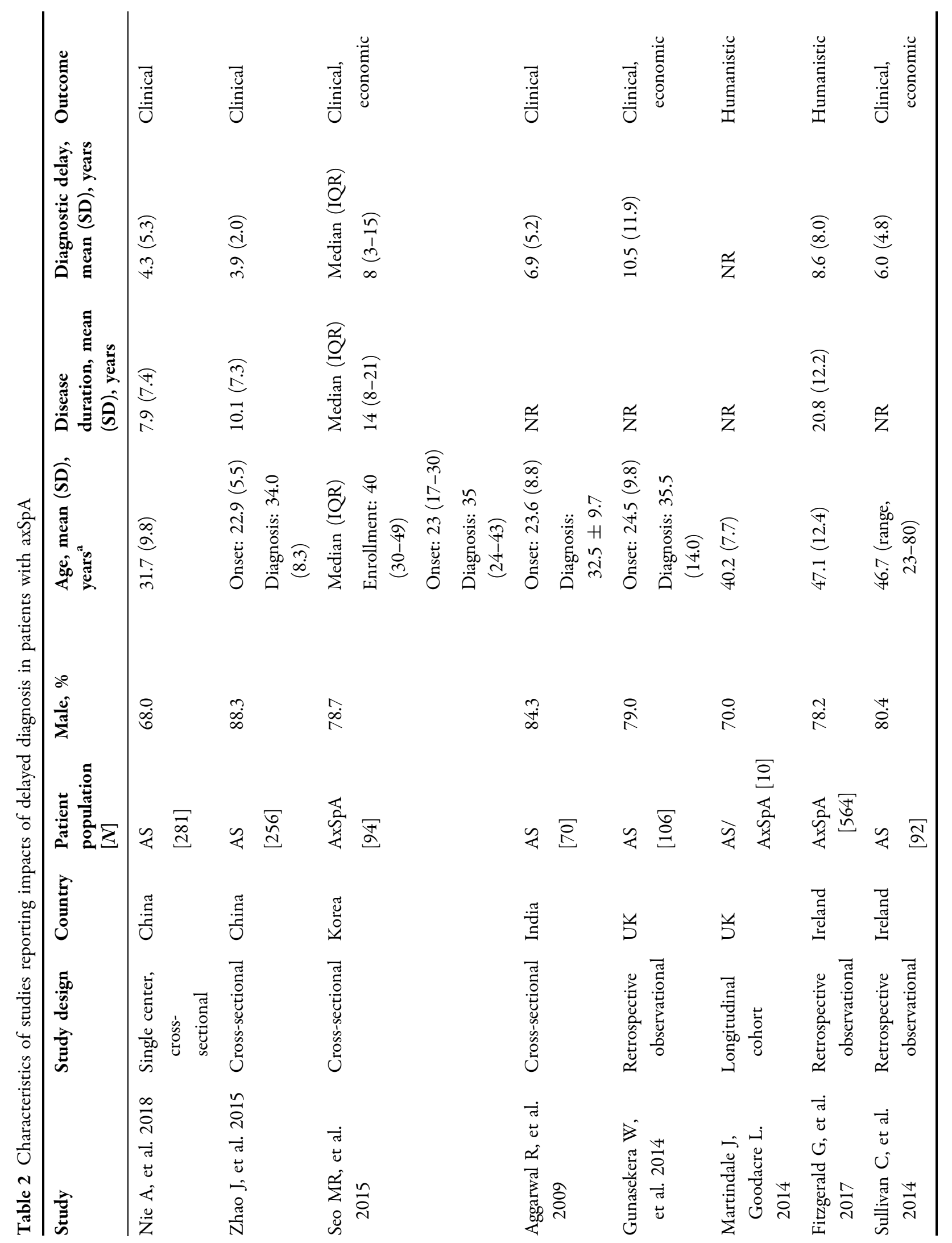




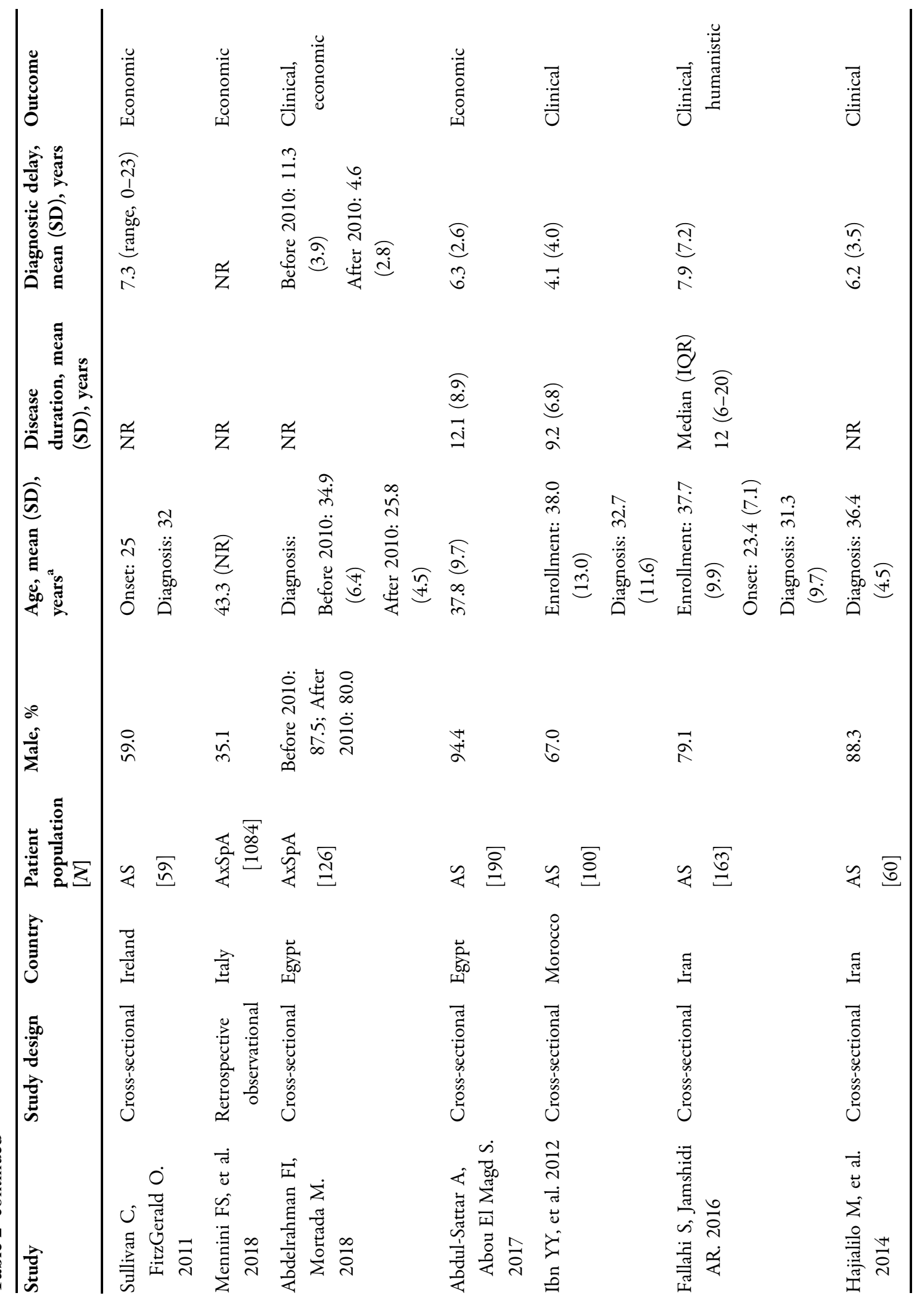




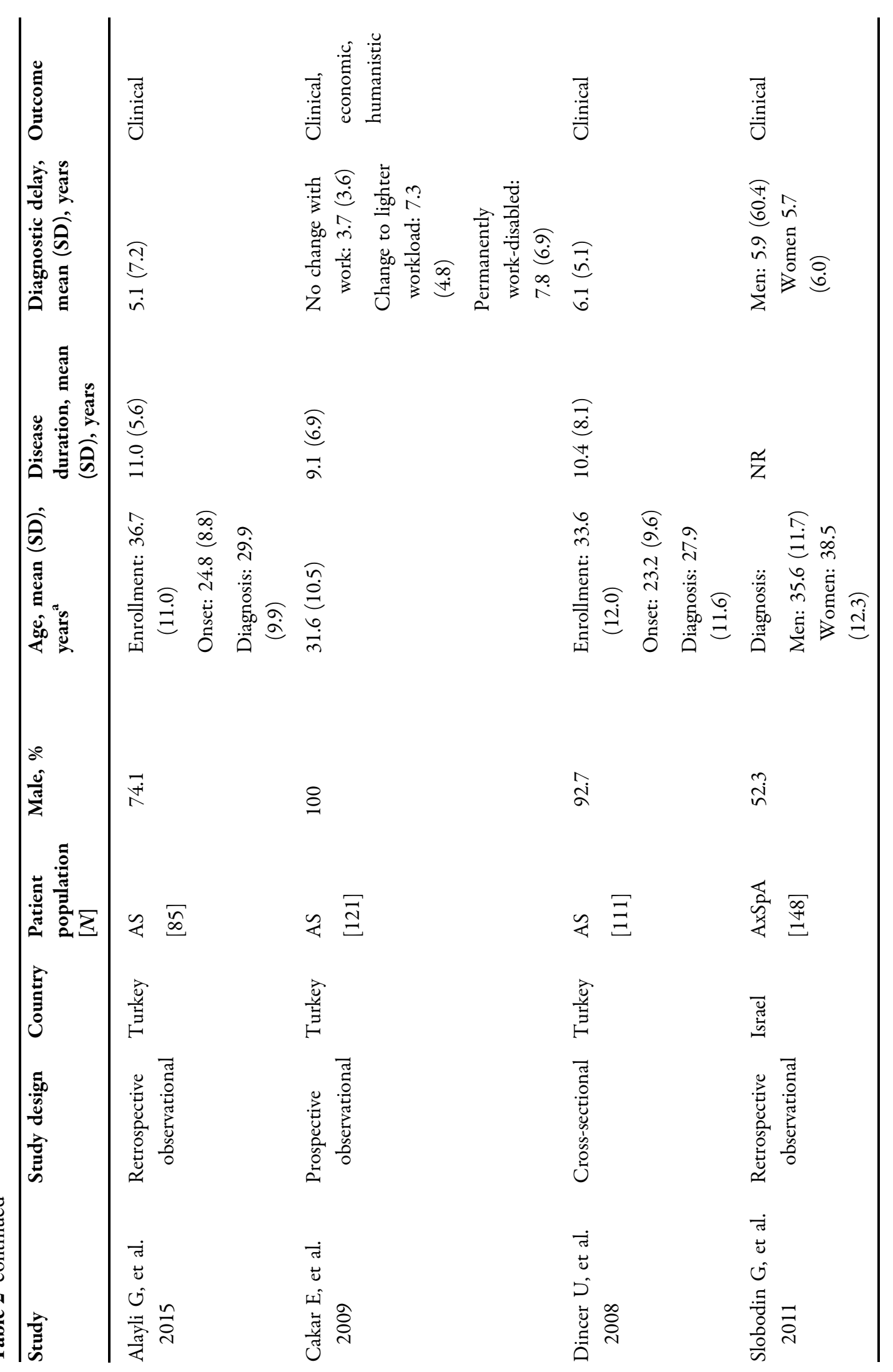




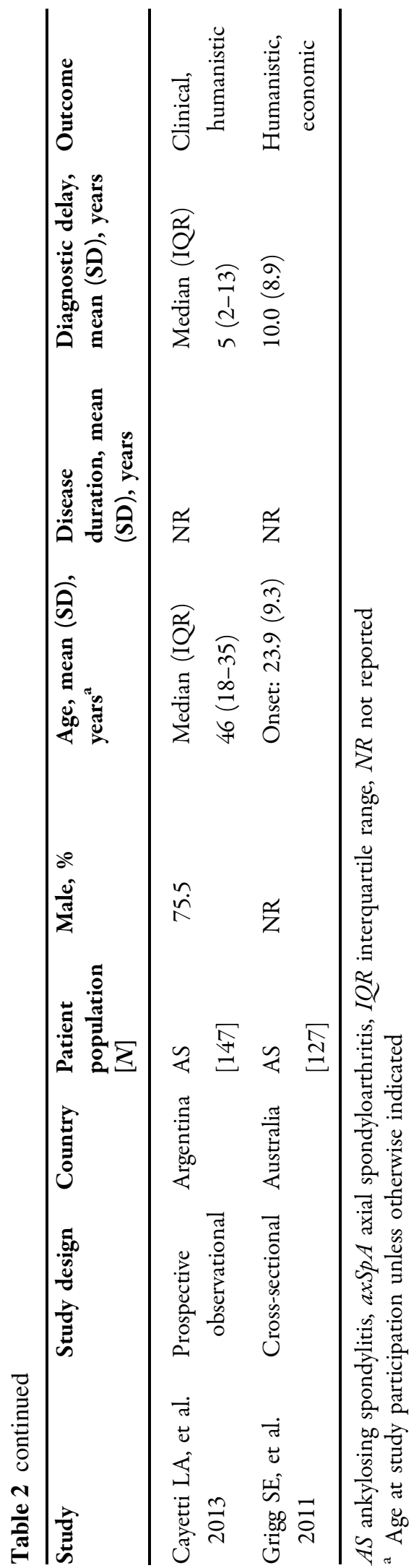

wall distance, lateral lumbar flexion); in all studies, patients with a longer diagnosis delay had worse spinal mobility than those with a shorter delay (Table 3) [6, 46, 59, 62, 64].

A total of six studies assessed radiographic structural progression, of which five found a significant association between longer diagnosis delay and greater radiographic progression $[6,46,50,59,61]$; in the remaining study, there was a trend toward greater radiographic progression in patients with longer diagnosis delay, but this difference did not reach statistical significance [65] (Table 3). In the two studies that evaluated mSASSS, patients with a longer diagnosis delay had significantly higher mSASSS than those with a shorter delay $[46,61]$; one study also found that patients with a longer delay had a higher mean change in mSASSS per year following diagnosis [46].

Four studies assessed CRP levels and ESR [46, 59, 62, 64] (Table 3). ESR was comparable between patients with early vs. late diagnosis in all studies. CRP levels were also comparable between patients with early vs. late diagnosis in three of four studies $[46,59,64]$; however, one study found that patients with a longer delay in diagnosis had higher CRP values than those with a shorter time to diagnosis [62].

\section{Economic Burden of Delayed Diagnosis}

The majority (six of nine) of studies that evaluated economic burden of delayed diagnosis assessed work disability or employment; in all of these studies, longer diagnosis delay was associated with a greater likelihood of work disability or unemployment [46, 52, 56, 58, 62, 66] (Table 4). In the three studies that evaluated treatment costs and healthcare utilization, longer diagnosis delay was associated with higher costs related to doctors' visits and specialist services $[35,57]$, unnecessary spinal surgery [35], and treatments [57, 66] (Table 4).

\section{Humanistic Burden of Delayed Diagnosis}

Five of six studies found that delayed diagnosis of axSpA was associated with negative impacts on health-related quality of life $[6,53,54,62,66]$; 
Table 3 Clinical impact of delayed diagnosis of axSpA

\begin{tabular}{|c|c|c|c|c|}
\hline Study & $\begin{array}{l}\text { Patient } \\
\text { population } \\
{[N]}\end{array}$ & $\begin{array}{l}\text { Definition of delay, } \\
\text { years }\end{array}$ & $\begin{array}{l}\text { Clinical } \\
\text { outcome } \\
\text { measures }\end{array}$ & Patient outcomes \\
\hline $\begin{array}{l}\text { Nie A, et al. } \\
2018\end{array}$ & $\begin{array}{l}\text { AS } \\
{[281]}\end{array}$ & Continuous & PSQI & $\begin{array}{l}\text { Longer delay significantly correlated with higher PSQI scores } \\
\quad(\beta=0.174 ; P=0.001)\end{array}$ \\
\hline $\begin{array}{l}\text { Zhao J, et al. } \\
\qquad 2015\end{array}$ & $\begin{array}{l}\text { AS } \\
{[256]}\end{array}$ & $\leq 5$ vs. $>5$ & BASRI-hip & $\begin{array}{l}\text { Delay of }>5 \text { years positively associated with more severe hip } \\
\text { disease (OR, 2.35 [95\% CI, 1.36-4.08]; } P=0.002)\end{array}$ \\
\hline $\begin{array}{l}\text { Seo MR, et al. } \\
2015\end{array}$ & $\begin{array}{l}\text { AxSpA } \\
{[94]}\end{array}$ & $\begin{array}{l}\leq 8 \text { (late diagnosis) } \\
\quad \text { vs. }>8 \text { (early } \\
\quad \text { diagnosis) }\end{array}$ & $\begin{array}{l}\text { CRP } \\
\text { ESR } \\
\text { BASDAI } \\
\text { BASFI } \\
\text { Modified } \\
\text { Schober test } \\
\text { Radiographic } \\
\text { sacroiliitis III } \\
\text { or IV } \\
\text { Spine bony } \\
\text { change } \\
\text { mSASSS } \\
\text { ACR } \\
\text { functional } \\
\text { class III or IV }\end{array}$ & $\begin{array}{l}\text { Significant differences between late vs. early diagnosis at time } \\
\text { of diagnosis: } \\
\text { Modified Schober test: median (IQR), } 2.7(1.6-4.4) \text { vs. } 6.0 \\
(2.5-6.0) \mathrm{cm} ; P=0.03 \\
\text { mSASSS: median (IQR), } 21.0(3.0-42.0) \text { vs. } 0(0-4.5) ; \\
P<0.01 \\
\text { Proportion with spine bony changes: } 77.4 \text { vs. } 44.8 \% \text {; } \\
P<0.01 \\
\text { Significant differences between late vs. early diagnosis at time } \\
\text { of study: } \\
\text { BASDAI: median (IQR), } 3.4(2.0-4.9) \text { vs. } 2.0(1.0-4.2) \text {; } \\
P=0.01 \\
\text { BASFI: median (IQR), } 2.5(0.3-3.8) \text { vs. } 0.7(0.1-1.4) \text {; } \\
P<0.01 \\
\text { Modified Schober test: median (IQR), } 2.8(1.2-4.5) \text { vs. } 4.5 \\
\quad(3.3-5.3) \text { cm; } P<0.01 \\
\text { mSASSS: median (IQR), } 26.0 \text { (4.8-46.3) vs. } 1.0(0-12.5) \text {; } \\
P<0.01 \\
\text { Proportion with spine bony changes: } 85.3 \text { vs. } 53.6 \% \text {; } \\
P<0.01\end{array}$ \\
\hline $\begin{array}{l}\text { Aggarwal R, } \\
\text { et al. } 2009\end{array}$ & $\begin{array}{l}\text { AS } \\
{[70]}\end{array}$ & $\leq 5.9$ vs. $>5.9$ & $\begin{array}{l}\text { BASDAI } \\
\text { BASFI } \\
\text { BASMI }\end{array}$ & $\begin{array}{l}\text { Patients with delay of }>5.9 \text { years had significantly worse } \\
\text { mean (SD) scores than those with delay of } \leq 5.9 \text { years } \\
\text { BASDAI: } 3.7(1.8) \text { vs. } 2.7(1.7) ; P=0.035 \\
\text { BASFI: } 3.8(2.4) \text { vs. } 2.5(2.1) ; P=0.033 \\
\text { BASMI: } 3.3(2.7) \text { vs. } 1.5(2.2) ; P=0.012\end{array}$ \\
\hline $\begin{array}{l}\text { Gunasekera W, } \\
\text { et al. } 2014\end{array}$ & AS [106] & Continuous & $\begin{array}{l}\text { BASDAI } \\
\text { BASFI } \\
\text { BASMI }\end{array}$ & $\begin{array}{l}\text { BASMI score increased by } 0.06 \text { per year of diagnosis delay } \\
\quad(P=0.0002) \\
\text { No significant impact of diagnosis delay on BASDAI or } \\
\text { BASFI }\end{array}$ \\
\hline $\begin{array}{l}\text { Sullivan C, et al. } \\
2014\end{array}$ & $\begin{array}{l}\text { AS } \\
{[92]}\end{array}$ & $\begin{array}{l}<4 \text { vs. } 5-9 \text { vs. }>10 \\
\text { vs. unknown }\end{array}$ & $\begin{array}{l}\text { BASDAI } \\
\text { BASFI } \\
\text { BASMI }\end{array}$ & $\begin{array}{l}\text { No significant differences in BASDAI, BASFI, or BASMI } \\
\text { scores }\end{array}$ \\
\hline $\begin{array}{l}\text { Abdelrahman } \\
\text { FI, Mortada } \\
\text { M. } 2018\end{array}$ & $\begin{array}{l}\text { AxSpA } \\
{[126]}\end{array}$ & $\begin{array}{l}\text { Mean (SD) Before } \\
\quad \text { 2010: } 11.3(3.9) \\
\text { After 2010: } \\
4.6(2.8)\end{array}$ & $\begin{array}{l}\text { BASDAI } \\
\text { BASFI } \\
\text { BASMI }\end{array}$ & $\begin{array}{l}\text { Patients with longer delay had worse mean }(\mathrm{SD}) \text { scores than } \\
\text { those with shorter delay (all } P<0.001) \text { : } \\
\text { BASDAI: } 9.1(1.4) \text { vs. } 4.3(2.2) \\
\text { BASFI: } 9.1(1.4) \text { vs. } 3.9(2.2) \\
\text { BASMI: } 8.9(1.2) \text { vs. } 2.2(2.0)\end{array}$ \\
\hline
\end{tabular}


Table 3 continued

\begin{tabular}{|c|c|c|c|c|}
\hline$\overline{\text { Study }}$ & $\begin{array}{l}\text { Patient } \\
\text { population } \\
{[N]}\end{array}$ & $\begin{array}{l}\text { Definition of delay, } \\
\text { years }\end{array}$ & $\begin{array}{l}\text { Clinical } \\
\text { outcome } \\
\text { measures }\end{array}$ & Patient outcomes \\
\hline $\begin{array}{l}\text { Ibn YY, et al. } \\
2012\end{array}$ & $\begin{array}{l}\text { AS } \\
{[100]}\end{array}$ & $<5$ vs. $\geq 5$ & $\begin{array}{l}\text { CRP } \\
\text { ESR } \\
\text { BASDAI } \\
\text { BASFI } \\
\text { BASRI } \\
\text { Occiput-to- } \\
\text { wall distance } \\
\text { Chest } \\
\text { expansion } \\
\text { Schober test }\end{array}$ & $\begin{array}{l}\text { Patients with delay of } \geq 5 \text { years had significantly greater } \\
\text { BASFI }(61.4 \text { vs. } 51.1) \text { and BASRI }(8.4 \text { vs. } 5.7) \text { scores and } \\
\text { significantly lower chest expansion }(2.2 \text { vs. } 3.5 \mathrm{~cm}) \text { and } \\
\text { Schober test result }(1.7 \text { vs. } 2.8 \mathrm{~cm}) \text { than those with delay } \\
\text { of }<5 \text { years (all } P<0.05) \\
\text { Longer diagnostic delay was significantly correlated with: } \\
\text { Occiput-to-wall distance: } r=0.317(P=0.001) \\
\text { Chest expansion: } r=0.374(P>0.001) \\
\text { Schober test: } r=-0.368(P<0.001) \\
\text { BASFI: } r=0.289(P=0.004) \\
\text { BASRI: } r=0.349(P<0.001) \\
\text { No impact of diagnostic delay on BASDAI score, ESR, or } \\
\text { CRP level }\end{array}$ \\
\hline $\begin{array}{l}\text { Fallahi S, } \\
\text { Jamshidi AR. } \\
2016\end{array}$ & $\begin{array}{l}\text { AS } \\
{[163]}\end{array}$ & Continuous & $\begin{array}{l}\text { BASDAI } \\
\text { BASFI } \\
\text { BASMI } \\
\text { Chest } \\
\text { expansion } \\
\text { Finger-to-floor } \\
\text { distance } \\
\text { Intermalleolar } \\
\text { distance } \\
\text { Modified } \\
\text { Schober test } \\
\text { Cervical } \\
\text { rotation } \\
\text { Tragus-to-wall } \\
\text { distance } \\
\text { Sacroiliitis } \\
\text { grading }\end{array}$ & $\begin{array}{l}\text { Longer diagnostic delay significantly correlated with worse } \\
\text { outcomes: } \\
\text { BASDAI: } r=0.18(P=0.026) \\
\text { BASFI: } r=0.23(P=0.003) \\
\text { BASMI: } r=0.41(P<0.001) \\
\text { Chest expansion: } r=-0.38(P<0.001) \\
\text { Finger-to-floor distance: } r=0.27(P<0.001) \\
\text { Intermalleolar distance: } r=-0.18(P=0.022) \\
\text { Modified Schober test: } r=-0.33(P<0.001) \\
\text { Cervical rotation: } r=-0.29(P<0.001) \\
\text { Tragus-to-wall distance: } r=0.30(P<0.01) \\
\text { Sacroiliitis grading: } r=0.16(P=0.042)\end{array}$ \\
\hline $\begin{array}{l}\text { Hajialilo M, } \\
\text { et al. } 2014\end{array}$ & $\begin{array}{l}\text { AS } \\
{[60]}\end{array}$ & $<3$ vs. $>3$ & $\begin{array}{l}\text { BASDAI } \\
\text { BASFI }\end{array}$ & $\begin{array}{l}\text { Patients with delay of }>3 \text { years had significantly worse BASFI } \\
\text { scores (mean [SD], } 4.1[0.7] \text { vs. } 3.3[1.0] ; P=0.001 \text { ) but } \\
\text { comparable BASDAI scores vs. patients with delay } \\
\text { of }<3 \text { years }\end{array}$ \\
\hline $\begin{array}{l}\text { Alayli } G \text {, et al. } \\
2015\end{array}$ & $\begin{array}{l}\text { AS } \\
{[85]}\end{array}$ & Continuous & $\begin{array}{l}\text { BASDAI } \\
\text { BASFI } \\
\text { BASMI } \\
\text { mSASSS }\end{array}$ & $\begin{array}{l}\text { Delayed diagnosis positively correlated with BASFI and } \\
\text { BASMI scores and mSASSS but not with BASDAI scores }\end{array}$ \\
\hline
\end{tabular}


Table 3 continued

\begin{tabular}{|c|c|c|c|c|}
\hline Study & $\begin{array}{l}\text { Patient } \\
\text { population } \\
{[N]}\end{array}$ & $\begin{array}{l}\text { Definition of delay, } \\
\text { years }\end{array}$ & $\begin{array}{l}\text { Clinical } \\
\text { outcome } \\
\text { measures }\end{array}$ & Patient outcomes \\
\hline $\begin{array}{l}\text { Cakar E, et al. } \\
2009\end{array}$ & $\begin{array}{l}\text { AS } \\
{[121]}\end{array}$ & $\begin{array}{l}\text { Mean (SD) } \\
\text { No change in work: } \\
3.7 \text { (3.6) } \\
\text { Work-disabled, } \\
\text { change in job: } 7.3 \\
(4.8) \\
\text { Permanently work } \\
\text { disabled: } 7.8(6.9)\end{array}$ & $\begin{array}{l}\text { CRP } \\
\text { ESR } \\
\text { BASFI } \\
\text { BASDAI } \\
\text { BASMI } \\
\text { Modified } \\
\text { lumbar } \\
\text { Schober test } \\
\text { Lateral lumbar } \\
\text { flexion } \\
\text { Chest } \\
\text { expansion } \\
\text { Chin-to- } \\
\text { sternum } \\
\text { distance } \\
\text { Tragus-to-wall } \\
\text { distance } \\
\text { Intermalleolar } \\
\text { distance }\end{array}$ & $\begin{array}{l}\text { Patients with longer delay had higher CRP levels and worse } \\
\text { mobility than those with shorter delay (mean [SD], no } \\
\text { change vs. job change vs. permanently disabled): } \\
\text { CRP, mg/L: } 11.1(8.3) \text { vs. } 17.6(22.2) \text { vs. } 39.7(46.4) ; \\
P=0.034 \\
\text { Modified lumbar Schober test, cm: } 4.3(1.4) \text { vs. } 3.2(1.3) \text { vs. } \\
2.0(1.5) ; P<0.001 \\
\text { Lateral lumbar flexion, cm: } 18.2(10.8) \text { vs. } 14.7(12.7) \text { vs. } 7.8 \\
(3.5) ; P<0.001 \\
\text { Chest expansion, cm: } 5.5(2.0) \text { vs. } 4.5(2.1) \text { vs. } 2.4(1.5) \text {; } \\
P<0.001 \\
\text { Chin-sternum distance, cm: } 0.2(0.8) \text { vs. } 1.3(1.8) \text { vs. } 2.0 \\
(2.4) ; P=0.001 \\
\text { Tragus-to-wall distance, cm: } 0.6(1.9) \text { vs. } 1.2(2.5) \text { vs. } 6.9 \\
(6.2) ; P=0.001 \\
\text { Intermalleolar distance, cm: } 113.9(15.3) \text { vs. } 105.3(17.2) \text { vs. } \\
\text { 90.6 (21.3); } P<0.001 \\
\text { BASFI: } 3.3(2.1) \text { vs. } 4.6(2.1) \text { vs. } 5.2(2.5) ; P=0.005 \\
\text { BASMI: } 1.1(1.1) \text { vs. } 2.4(1.3) \text { vs. } 4.3(2.3) ; P<0.001\end{array}$ \\
\hline $\begin{array}{l}\text { Dincer U, et al. } \\
2008\end{array}$ & $\begin{array}{l}\text { AS } \\
{[111]}\end{array}$ & $\begin{array}{l}<3 \text { years vs. } \\
>3 \text { years }\end{array}$ & $\begin{array}{l}\text { BASDAI } \\
\text { BASFI }\end{array}$ & No significant differences in BASDAI or BASFI scores \\
\hline $\begin{array}{l}\text { Slobodin G, } \\
\text { et al. } 2011\end{array}$ & $\begin{array}{l}\text { AxSpA } \\
{[148]}\end{array}$ & $\leq 1$ vs. $1-5$ vs. $\geq 5$ & $\begin{array}{l}\text { CRP } \\
\text { ESR } \\
\text { Schober test } \\
\text { Finger-to-floor } \\
\text { distance } \\
\text { Occiput-to- } \\
\text { wall distance } \\
\text { Chest } \\
\text { expansion }\end{array}$ & $\begin{array}{l}\text { Patients with delay of }>1 \text { year had significantly }(P=0.028) \\
\text { greater occiput-to-wall distance (less spinal flexibility) than } \\
\text { those diagnosed within } 1 \text { year (mean }[S D]: \leq 1 \text { year, } 1.5 \\
[5.4] \mathrm{cm} ; 1-5 \text { years, } 3.7[2.0] \mathrm{cm} ; \geq 5 \text { years, } 2.4[5.3])\end{array}$ \\
\hline $\begin{array}{l}\text { Cayetti LA, } \\
\text { et al. } 2013\end{array}$ & $\begin{array}{l}\text { AS } \\
{[147]}\end{array}$ & $\begin{array}{l}\leq 3 \text { vs. }>3 \\
\text { to } \leq 10 \text { vs. }>10\end{array}$ & $\begin{array}{l}\text { BASDAI } \\
\text { BASFI } \\
\text { BASMI } \\
\text { BASRI }\end{array}$ & $\begin{array}{l}\text { No substantial impact of delay on functional capacity or } \\
\text { radiographic damage }\end{array}$ \\
\hline
\end{tabular}

$A C R$ American College of Rheumatology, $A S$ ankylosing spondylitis, axSpA axial spondyloarthritis, $B A S D A I$ Bath Ankylosing Spondylitis Disease Activity Index, BASFI Bath Ankylosing Spondylitis Functional Index, BASMI Bath Ankylosing Spondylitis Metrology Index, BASRI Bath Ankylosing Spondylitis Radiology Index, CRP C-reactive protein, ESR erythrocyte sedimentation rate, IQR interquartile range, $m S A S S S$ modified Stoke Ankylosing Spondylitis Spinal Score, PSQI Pittsburgh Sleep Quality Index

however, results for specific outcome measures varied across studies (Table 5). Although one study found a significantly higher prevalence of physician-reported depression among patients with a diagnosis delay of $\geq 7$ years among than those with a delay of $<7$ years [54], two studies found no significant association between longer diagnosis delay and Beck Depression Inventory scores $[62,66]$. Similarly, one study showed that longer diagnosis delay was significantly correlated 
Table 4 Economic impacts of delayed diagnosis of axSpA

\begin{tabular}{|c|c|c|c|c|}
\hline Study & $\begin{array}{l}\text { Patient } \\
\text { population } \\
{[N]}\end{array}$ & $\begin{array}{l}\text { Definition of } \\
\text { delay, years }\end{array}$ & $\begin{array}{l}\text { Economic outcome } \\
\text { measures }\end{array}$ & Patient outcomes \\
\hline $\begin{array}{l}\text { Seo MR, et al. } \\
2015\end{array}$ & $\begin{array}{l}\text { SpA } \\
{[105]}\end{array}$ & $\leq 8$ vs. $>8$ & Social disabilities ${ }^{a}$ & $\begin{array}{l}\text { Higher proportion of patients with delay } \\
\text { of }>8 \text { years reported social disabilities } \\
\text { compared with those with delay of } \leq 8 \text { years, } \\
\text { although the difference did not reach statistical } \\
\text { significance }(28.3 \text { vs. } 12.8 \% ; P=0.06)\end{array}$ \\
\hline $\begin{array}{l}\text { Gunasekera W, } \\
\text { et al. } 2014\end{array}$ & $\begin{array}{l}\text { AS } \\
{[106]}\end{array}$ & Continuous & Work disability & $\begin{array}{l}\text { Patients who were work disabled had a } \\
\text { significantly longer delay than those who were } \\
\text { not work disabled (mean, } 16.6 \text { vs. } 7.8 \text { years; } \\
\quad P=0.005 \text { ) } \\
\text { Risk of being work disabled increased by } 6.6 \% \\
\text { per year of delay (OR, } 1.07 \text { [CI, } 1.0-1.1] \text {; } \\
P=0.0009 \text { ) }\end{array}$ \\
\hline $\begin{array}{l}\text { Sullivan C, } \\
\text { et al. } 2014\end{array}$ & $\begin{array}{l}\text { AS } \\
{[92]}\end{array}$ & $\begin{array}{c}0-4 \text { vs. } 5-9 \\
\text { vs. }>10 \text { vs. } \\
\text { unknown }\end{array}$ & TNFi use & $\begin{array}{l}\text { No relationship between diagnostic delay and } \\
\text { likelihood of TNFi use }\end{array}$ \\
\hline $\begin{array}{l}\text { Sullivan C, } \\
\text { FitzGerald O. } \\
2011\end{array}$ & $\begin{array}{l}\text { AS } \\
{[59]}\end{array}$ & $\begin{array}{l}<4 \text { vs. } 5-9 \\
\text { vs. }>10\end{array}$ & Employment & $\begin{array}{l}\text { Longer delay was associated with greater } \\
\text { likelihood of work disability; unemployment } \\
\text { rose from } 20 \text { to } 29 \% \text { to } 41 \% \text { among those } \\
\text { diagnosed in }<4 \text { years, } 5-9 \text { years, } \\
\text { and }>10 \text { years, respectively }\end{array}$ \\
\hline $\begin{array}{r}\text { Mennini FS, } \\
\text { et al. } 2018\end{array}$ & $\begin{array}{l}\text { AxSpA } \\
{[1084]}\end{array}$ & $\begin{array}{l}3 \text { years prior } \\
\text { to initial } \\
\text { SpA } \\
\text { diagnosis }\end{array}$ & $\begin{array}{l}\text { Cost }(€) \text { of SpA- } \\
\text { related specialist } \\
\text { visits and } \\
\text { treatments }\end{array}$ & $\begin{array}{l}\text { In } 3 \text { years prior to } \mathrm{SpA} \text { diagnosis, patients } \\
\text { received an average of } 4 \text { specialist services and } \\
4 \text { treatments related to undiagnosed } \mathrm{SpA}, \\
\text { resulting in an average cost of } \approx € 140.90 \text { per } \\
\text { patient, corresponding to } \approx € 152,767 \text { for } \\
\text { study population and } \approx € 5,387,972 \text { for } \\
\text { Italian population of patients with } \mathrm{SpA}\end{array}$ \\
\hline $\begin{array}{l}\text { Abdelrahman } \\
\text { FI, Mortada } \\
\text { M. } 2018\end{array}$ & $\begin{array}{l}\text { AxSpA } \\
{[126]}\end{array}$ & $\begin{array}{l}\text { Mean }(\mathrm{SD}) \\
11.3(3.9) \\
\text { vs. } \\
4.6(2.8)\end{array}$ & $\begin{array}{l}\text { Healthcare costs } \\
\text { Doctor visits } \\
\text { Spinal surgery }\end{array}$ & $\begin{array}{l}\text { Patients with longer delay had worse economic } \\
\text { outcomes than those with shorter delay (all } \\
P<0.001) \text { : } \\
\text { Cost of delay period, mean }(\mathrm{SD}): \$ 9879.30 \\
(\$ 3827.20) \text { vs. } \$ 2373.90(\$ 881.80) \\
\text { No. of doctor visits during delay period, mean } \\
(\mathrm{SD}): 14.3(6) \text { vs. } 5.6(3.4) \\
\text { Proportion of patients with unnecessary spinal } \\
\text { surgery: } 65.4 \text { vs. } 34.6 \%\end{array}$ \\
\hline
\end{tabular}


Table 4 continued

\begin{tabular}{|c|c|c|c|c|}
\hline Study & $\begin{array}{l}\text { Patient } \\
\text { population } \\
{[N]}\end{array}$ & $\begin{array}{l}\text { Definition of } \\
\text { delay, years }\end{array}$ & $\begin{array}{l}\text { Economic outcome } \\
\text { measures }\end{array}$ & Patient outcomes \\
\hline $\begin{array}{l}\text { Abdul-Sattar A, } \\
\text { Abou El } \\
\text { Magd S. } 2017\end{array}$ & $\begin{array}{l}\text { AS } \\
{[190]}\end{array}$ & Continuous & Work disability & $\begin{array}{l}\text { Patients who were work disabled had } \\
\text { significantly longer delay than those who were } \\
\text { not work disabled (mean }[\mathrm{SD}], 8[2.9] \text { vs. } 4 \\
\text { [2.1] years; } P<0.001 \text { ) } \\
\text { Longer delay was associated with greater } \\
\text { likelihood of work disability (OR, } 2.1[95 \% \mathrm{CI} \text {, } \\
\text { 1.00-3.40]; } P=0.001 \text { ) }\end{array}$ \\
\hline $\begin{array}{l}\text { Cakar E, et al. } \\
2009\end{array}$ & $\begin{array}{l}\text { AS } \\
{[121]}\end{array}$ & Continuous & Work disability & $\begin{array}{l}\text { Patients who were permanently work disabled or } \\
\text { changed jobs due to work disability had longer } \\
\text { mean }(\mathrm{SD}) \text { delay }(7.8[6.9] \text { and } 7.3[4.8] \text { years) } \\
\text { than those who were not work disabled (3.7 } \\
[3.6] \text { years; } P=0.028)\end{array}$ \\
\hline $\begin{array}{l}\text { Grigg SE, et al. } \\
2011\end{array}$ & $\begin{array}{l}\text { AS } \\
{[127]}\end{array}$ & $\begin{array}{l}<5 \text { vs. } 5-10 \\
\text { vs. }>10\end{array}$ & $\begin{array}{l}\text { Treatment costs }(\$) \\
\text { Employability }\end{array}$ & $\begin{array}{l}\text { Estimated cost of treatment prior to diagnosis } \\
\text { was }>\$ 3000 \text { in } 25.6 \% \text { of patients } \\
\text { with }<5 \text { years of delay vs. } 44.4 \% \text { with } \\
5-10 \text { years of delay }(P=0.08) \text { and } 67.4 \% \\
\text { with }>10 \text { years of delay }(P=0.002) \\
\text { Employability was affected in } 66.7 \% \text { of patients } \\
\text { with }<5 \text { years of delay vs. } 75.6 \% \text { with } \\
5-10 \text { years of delay }(P=0.37) \text { and } 90.7 \% \\
\text { with }>10 \text { years of delay }(P=0.003)\end{array}$ \\
\hline
\end{tabular}

$A S$ ankylosing spondylitis, axSpA axial spondyloarthritis, $O R$ odds ratio, $S p A$ spondyloarthritis, $T N F i$ tumor necrosis factor inhibitor

a Defined as job changes or the complete discontinuation of work attributable to the disease among workers, discontinued studies among students, and requiring the assistance of another person among homemakers

with worse ASQOL scores and more morning stiffness [6], whereas two studies showed no significant difference in ASQOL [65] or morning stiffness [62] between patients with a late vs. early diagnosis. Humanistic burden was assessed using the 36-item Short Form Health Survey in one study, which showed that longer diagnosis delay was associated with worse scores in the physical functioning and general health domains [62]. In one study, patients reported experiencing negative psychological impacts during the delay period [53]; in a separate study, patients reported feeling emotional relief and a more positive outlook regarding the disease once they received a diagnosis [66].

\section{DISCUSSION}

A limited number of studies have evaluated the impact of delayed diagnosis on disease burden in patients with axSpA, particularly with respect to economic and humanistic outcomes. The included studies were conducted in only 13 countries, and only six countries had $\geq 1$ study. Prevalence of axSpA and application of classification criteria in routine practice vary by 
Table 5 Humanistic impacts of delayed diagnosis of axSpA

\begin{tabular}{|c|c|c|c|c|}
\hline Study & $\begin{array}{l}\text { Patient } \\
\text { population } \\
{[N]}\end{array}$ & $\begin{array}{l}\text { Definition of } \\
\text { delay, years }\end{array}$ & $\begin{array}{l}\text { Humanistic } \\
\text { outcome } \\
\text { measures }\end{array}$ & Patient outcomes \\
\hline $\begin{array}{l}\text { Martindale J, } \\
\text { Goodacre L. } \\
2014\end{array}$ & $\begin{array}{l}\text { AS/AxSpA } \\
{[10]}\end{array}$ & Continuous & $\begin{array}{l}\text { Emotional and } \\
\text { social health }\end{array}$ & $\begin{array}{l}\text { In the period between symptom onset and diagnosis (mean [SD], } \\
10.1 \text { [7.3] years; range, } 1-20 \text { years), patients experienced } \\
\text { negative psychological impact, including desperation, distress, } \\
\text { depression, and feeling disheartened; employed patients felt } \\
\text { stigmatized by the perception of a "bad back" }\end{array}$ \\
\hline $\begin{array}{l}\text { Fitzgerald G, } \\
\text { et al. } 2017\end{array}$ & $\begin{array}{l}\mathrm{AxSpA} \\
{[564]}\end{array}$ & $<7$ vs. $\geq 7$ & Depression & $\begin{array}{l}\text { Higher prevalence of depression in patients with delay } \\
\text { of } \geq 7 \text { years than those with delay of }<7 \text { years }(15.5 \text { vs. } \\
9.1 \% ; P=0.032)\end{array}$ \\
\hline $\begin{array}{l}\text { Fallahi S, } \\
\text { Jamshidi } \\
\text { AR. } 2016\end{array}$ & $\begin{array}{l}\text { AS } \\
{[163]}\end{array}$ & Continuous & $\begin{array}{l}\text { ASQOL } \\
\text { Fatigue }^{\mathrm{a}} \\
\text { Morning } \\
\text { stiffness }^{\mathrm{b}}\end{array}$ & $\begin{array}{l}\text { Delay positively correlated with worse ASQOL scores }(r=0.21 \text {; } \\
\quad P=0.008) \text { and morning stiffness }(r=0.21 ; P=0.006)\end{array}$ \\
\hline $\begin{array}{l}\text { Cakar E, et al. } \\
2009\end{array}$ & $\begin{array}{l}\text { AS } \\
{[121]}\end{array}$ & $\begin{array}{l}\text { Mean (SD) } \\
\text { No change in } \\
\text { work: } 3.7(3.6) \\
\text { Work disabled, } \\
\text { change in job: } \\
7.3(4.8) \\
\text { Permanently work } \\
\text { disabled: } 7.8 \\
(6.9)\end{array}$ & $\begin{array}{l}\text { Morning } \\
\quad \text { stiffness } \\
\text { BAS-G } \\
\text { SF-36 } \\
\text { Beck } \\
\text { Depression } \\
\text { Inventory }\end{array}$ & $\begin{array}{l}\text { Patients with longer delay had worse mean (SD) scores in } \\
\text { physical functioning and general health domains of SF-36 } \\
\text { than those with shorter delay } \\
\text { Physical functioning: no change, } 59.96(21.99) \text {; change in job, } \\
43.14 \text { (23.15); permanently disabled, } 51.16(19.95) ; P=0.009 \\
\text { General health: no change, } 46.00(20.90) \text {; change in job, } 33.67 \\
\text { (20.27); permanently disabled, } 22.04(19.99) ; P=0.035\end{array}$ \\
\hline $\begin{array}{l}\text { Cayetti LA, } \\
\text { et al. } 2013\end{array}$ & $\begin{array}{l}\text { AS } \\
{[147]}\end{array}$ & $\begin{aligned} & \leq 3 \text { vs. }>3 \\
& \text { to } \leq 10 \\
& \text { vs. }>10\end{aligned}$ & ASQOL & No significant differences in ASQOL scores between groups \\
\hline $\begin{array}{l}\text { Grigg SE, et al. } \\
2011\end{array}$ & $\begin{array}{l}\text { AS } \\
{[127]}\end{array}$ & $\begin{array}{l}<5 \text { vs. } 5-10 \\
\text { vs. }>10 \text { years }\end{array}$ & $\begin{array}{l}\text { Emotional } \\
\text { relief } \\
\text { Perception of } \\
\text { symptoms } \\
\text { Outlook for } \\
\text { the future } \\
\text { Beck } \\
\text { Depression } \\
\text { Inventory }\end{array}$ & $\begin{array}{l}\text { Once diagnosed, } 69 \% \text { experienced emotional relief, } 76 \% \\
\text { experienced a positive shift in perception of symptoms, and } \\
66 \% \text { had an optimistic outlook for the future } \\
\text { Delay was not associated with long-term depressed mood as } \\
\text { assessed by Beck Depression Inventory scores }\end{array}$ \\
\hline
\end{tabular}

$A S$ ankylosing spondylitis, $A S Q O L$ Ankylosing Spondylitis Quality of Life questionnaire, axSpA axial spondyloarthritis, $B A S-G$ Bath Ankylosing Spondylitis Patient Global Score, SF-36 Short Form 36

${ }^{a}$ BASDAI question 1, visual analog scale $0-10$

b BASDAI question 5, visual analog scale $0-10$

country [20-22, 43], which may influence length and burden of delay. Further studies are needed to gain a more comprehensive understanding of factors contributing to diagnosis delay and the impact of delayed diagnosis on disease burden globally and across a greater variety of practice settings.

Most of the identified studies included $<200$ patients, and the majority of patients across all studies were male and had a diagnosis of AS. Historically, axSpA has been considered a 
disease predominantly affecting males [67, 68], in part due to the perception of AS as the prototypical disease state $[21,25,30]$. The prevalence of AS is approximately 2- to 3-fold higher in men than women $[67,68]$. Definitive radiographic sacroiliitis and spinal damage occur less frequently in women than men [64, 69-71], and women are more likely to have peripheral symptoms [71-75] and extra-articular manifestations [71, 76-78]. Thus, early classification criteria that focus primarily on axial symptoms would be less likely to identify female patients with axSpA. The adoption of a broader definition of axSpA that includes peripheral features has revealed that the prevalence of axSpA may be nearly equal between men and women $[31,79,80]$ and that nonradiographic axSpA may be more common in women than men [81]. Based on the available literature, there is limited information on the impact of delayed diagnosis in women or patients with nonradiographic axSpA. More research is needed to better characterize the impact of diagnosis delay on overall disease burden in these populations.

Nevertheless, the available literature indicates that delayed diagnosis of axSpA is associated with an increased burden of disease, particularly with respect to mobility and function. The majority of studies that evaluated functional limitation and spinal mobility (e.g., BASFI, BASMI) found that longer diagnosis delay was associated with worse outcomes. Similarly, most studies that assessed radiographic structural progression showed that patients with a longer delay had more radiographic damage than those with shorter time to diagnosis. Previous studies have shown that radiographic damage is correlated with physical function and spinal mobility in patients with axSpA [82-85]. Therefore, there is a need for earlier diagnosis and treatment to prevent irreversible structural damage, which may in turn result in reduced mobility and greater functional limitation.

With respect to economic burden, the majority of the available literature focused on employment and work disability. Across all included studies, longer diagnosis delay was associated with more work disability and higher rates of job changes and unemployment, which may reflect the reduced mobility and greater functional impairment in patients with a longer delay. Three studies found that delayed diagnosis of axSpA resulted in increased healthcare costs and utilization. These studies were conducted in Italy [57], Egypt [35], and Australia [66], and two were single-center studies $[35,66]$; thus, further research is needed to understand the impact of diagnosis delay on costs and resource utilization in a broader range of healthcare settings.

Overall, the available data suggest that delayed diagnosis of axSpA is associated with an increased humanistic burden. Although conflicting results were observed across studies for specific outcomes such as ASQOL scores and depression, $\leq 3$ studies assessed any specific outcome measure, which limits the discernment of meaningful trends for any outcome. However, the majority of studies that assessed humanistic burden reported a negative impact of delayed diagnosis on $\geq 1$ measure of healthrelated quality of life, indicating that earlier diagnosis may alleviate some of the humanistic burden of axSpA.

These findings highlight the importance of comprehensively evaluating the impact of a delay in axSpA diagnosis and its potential impact on the healthcare system. Available data from this review demonstrated that earlier diagnosis and treatment led to better management of patient outcomes and healthcare costs. More research is needed to translate the clinical and psychological effects of untreated disease and misdiagnosis on healthcare resources. Education on classification criteria to distinguish key symptoms of axSpA and the development of diagnostic criteria that can be easily and routinely applied in clinical practice may lead to timely referrals, early diagnosis, and appropriate treatment to prevent irreversible damage that may impact every aspect of a patient's life.

\section{Limitations}

The definition of "delay" was widely variable among the studies, which may contribute to the differences in the impact of diagnosis delay on specific outcomes across studies. Because most of the studies included in this review were conducted across Europe and Asia, results may 
not be representative of all patients or healthcare systems. The majority of the studies included primarily male patients and therefore did not capture the longer delays typically experienced by female patients or account for other sex differences [86-92]. Additionally, the outcome measures used to assess economic and humanistic burden varied among the included studies. Together with the limited number of relevant studies and small patient population, these limitations precluded meaningful metaanalysis for the outcome measures assessed; thus, the results of this systematic literature review are descriptive in nature.

\section{CONCLUSIONS}

Overall, delayed diagnosis in patients with axSpA was associated with decreased physical function, higher direct and indirect costs, and negative psychological impacts. This study highlights the importance of early diagnosis and treatment of axSpA to improve outcomes and mitigate extensive burden on patients and society. Further efforts by the healthcare community are warranted to increase awareness of early signs of disease and reduce the delay in diagnosis of axSpA.

\section{ACKNOWLEDGEMENTS}

Funding. This study and the Rapid Service Fee was funded by Novartis Pharmaceuticals Corporation, East Hanover, NJ, USA.

Medical Writing and Editorial Assistance. Support for third-party writing assistance for this manuscript, furnished by Elizabeth Ohneck, PhD, of Health Interactions, Inc, was provided by Novartis Pharmaceuticals Corporation.

Authorship. All named authors meet the International Committee of Medical Journal Editors criteria for authorship for this article, take responsibility for the integrity of the work as a whole, and have given their approval for this version to be published.

Prior Presentation. A portion of these data were presented at the European League Against Rheumatism Annual European Congress of Rheumatology; June 12-15, 2019; Madrid, Spain.

Disclosures. Esther Yi and Yujin Park are employees of Novartis Pharmaceuticals Corporation. Amit Ahuja, Tanvi Rajput, and Aneesh Thomas George are employees of Novartis Healthcare Pvt Ltd.

Compliance with Ethics Guidelines. This article is based on previously conducted studies and does not contain any studies with human participants or animals performed by any of the authors.

Data Availability. All data generated or analyzed during this study are available in this published article or as supplementary information files.

Open Access. This article is distributed under the terms of the Creative Commons Attribution-NonCommercial 4.0 International License (http://creativecommons.org/licenses/ by-nc/4.0/), which permits any noncommercial use, distribution, and reproduction in any medium, provided you give appropriate credit to the original author(s) and the source, provide a link to the Creative Commons license, and indicate if changes were made.

\section{REFERENCES}

1. Sieper J, Poddubnyy D. Axial spondyloarthritis. Lancet. 2017;390:73-84.

2. Erol K, Gok K, Cengiz G, Kilic G, Kilic E, Ozgocmen S. Extra-articular manifestations and burden of disease in patients with radiographic and non-radiographic axial spondyloarthritis. Acta Reumatol Port. 2018;43:32-9. 
3. Strand V, Singh JA. Patient burden of axial spondyloarthritis. J Clin Rheumatol. 2017;23: 383-91.

4. Claudepierre P, Fagnani F, Cukierman G, et al. Burden of severe spondyloarthritis in France: a nationwide assessment of prevalence, associated comorbidities and cost. Joint Bone Spine. 2019;86: 69-75.

5. Nikiphorou E, Ramiro S, van der Heijde D, et al. Association of comorbidities in spondyloarthritis with poor function, work disability, and quality of life: results from the assessment of SpondyloArthritis international Society Comorbidities in Spondyloarthritis study. Arthritis Care Res (Hoboken). 2018;70:1257-62.

6. Fallahi S, Jamshidi AR. Diagnostic delay in ankylosing spondylitis: related factors and prognostic outcomes. Arch Rheumatol. 2015;31:24-30.

7. Deodhar A, Mease PJ, Reveille JD, et al. Frequency of axial spondyloarthritis diagnosis among patients seen by United States rheumatologists for evaluation of chronic back pain. Arthritis Rheumatol. 2016;68:1669-76.

8. Sykes MP, Doll H, Sengupta R, Gaffney K. Delay to diagnosis in axial spondyloarthritis: are we improving in the UK? Rheumatology (Oxford). 2015;54:2283-4.

9. Masson Behar V, Dougados M, Etcheto A, et al. Diagnostic delay in axial spondyloarthritis: a crosssectional study of 432 patients. Joint Bone Spine. 2017;84:467-71.

10. Redeker I, Callhoff J, Hoffmann F, et al. Determinants of diagnostic delay in axial spondyloarthritis: an analysis based on linked claims and patient-reported survey data. Rheumatology (Oxford). 2019;58:1634-8.

11. Garrido-Cumbrera M, Poddubnyy D, Gossec L, et al. The European map of axial spondyloarthritis: capturing the patient perspective-an analysis of 2846 patients across 13 countries. Curr Rheumatol Rep. 2019;21:19.

12. Strand V, Singh JA. Evaluation and management of the patient with suspected inflammatory spine disease. Mayo Clin Proc. 2017;92:555-64.

13. Proft F, Poddubnyy D. Ankylosing spondylitis and axial spondyloarthritis: recent insights and impact of new classification criteria. Ther Adv Musculoskelet Dis. 2018;10:129-39.

14. Hoy D, March L, Brooks P, et al. The global burden of low back pain: estimates from the Global Burden of Disease 2010 study. Ann Rheum Dis. 2014;73: 968-74.

15. Hoy D, Bain C, Williams G, et al. A systematic review of the global prevalence of low back pain. Arthritis Rheum. 2012;64:2028-37.

16. Manchikanti L, Singh V, Datta S, Cohen SP, Hirsch JA, American Society of Interventional Pain Physicians. Comprehensive review of epidemiology, scope, and impact of spinal pain. Pain Physician. 2009;12:E35-70.

17. Chou R, Qaseem A, Snow V, et al. Diagnosis and treatment of low back pain: a joint clinical practice guideline from the American College of Physicians and the American Pain Society. Ann Intern Med. 2007;147:478-91.

18. Braun J, Baraliakos X, Buehring B, Kiltz U, Fruth M. Imaging of axial spondyloarthritis. New aspects and differential diagnoses. Clin Exp Rheumatol. 2018;36(Suppl 114):35-42.

19. Meucci RD, Fassa AG, Faria NM. Prevalence of chronic low back pain: systematic review. Rev Saude Publica. 2015;49:73.

20. Stolwijk C, van Onna M, Boonen A, van Tubergen A. Global prevalence of spondyloarthritis: a systematic review and meta-regression analysis. Arthritis Care Res (Hoboken). 2016;68:1320-31.

21. Bohn R, Cooney M, Deodhar A, Curtis JR, Golembesky A. Incidence and prevalence of axial spondyloarthritis: methodologic challenges and gaps in the literature. Clin Exp Rheumatol. 2018;36: 263-74.

22. Wang R, Ward MM. Epidemiology of axial spondyloarthritis: an update. Curr Opin Rheumatol. 2018;30:137-43.

23. Kiltz U, Baraliakos X, Regel A, Buhring B, Braun J. Causes of pain in patients with axial spondyloarthritis. Clin Exp Rheumatol. 2017;35(Suppl 107):102-7.

24. Moll JM, Wright V. New York clinical criteria for ankylosing spondylitis. A statistical evaluation. Ann Rheum Dis. 1973;32:354-63.

25. van der Linden S, Valkenburg HA, Cats A. Evaluation of diagnostic criteria for ankylosing spondylitis. A proposal for modification of the New York criteria. Arthritis Rheum. 1984;27:361-8.

26. Poddubnyy D, Rudwaleit M, Haibel H, et al. Rates and predictors of radiographic sacroiliitis progression over 2 years in patients with axial spondyloarthritis. Ann Rheum Dis. 2011;70:1369-74. 
27. Wang R, Gabriel SE, Ward MM. Progression of nonradiographic axial spondyloarthritis to ankylosing spondylitis: a population-based cohort study. Arthritis Rheumatol. 2016;68:1415-21.

28. Amor B, Dougados M. Mijiyawa M [Criteria of the classification of spondylarthropathies]. Rev Rhum Mal Osteoartic. 1990;57:85-9.

29. Dougados $M$, van der Linden $S$, Juhlin $R$, et al. The European Spondylarthropathy Study Group preliminary criteria for the classification of spondylarthropathy. Arthritis Rheum. 1991;34:1218-27.

30. Rudwaleit M, Landewe R, van der Heijde D, et al. The development of assessment of SpondyloArthritis international Society classification criteria for axial spondyloarthritis (part I): classification of paper patients by expert opinion including uncertainty appraisal. Ann Rheum Dis. 2009;68:770-6.

31. Rudwaleit M, van der Heijde D, Landewe R, et al. The development of asssessment of SpondyloArthritis International Society classification criteria for axial spondyloarthritis (part II): validation and final selection. Ann Rheum Dis. 2009;68: 777-83.

32. Strand V, Rao SA, Shillington AC, Cifaldi MA, McGuire M, Ruderman EM. Prevalence of axial spondyloarthritis in United States rheumatology practices: assessment of SpondyloArthritis International Society criteria versus rheumatology expert clinical diagnosis. Arthritis Care Res (Hoboken). 2013;65:1299-306.

33. Salvadorini G, Bandinelli F, Delle Sedie A, et al. Ankylosing spondylitis: how diagnostic and therapeutic delay have changed over the last six decades. Clin Exp Rheumatol. 2012;30:561-5.

34. Sørensen J, Hetland ML, all departments of rheumatology in Denmark. Diagnostic delay in patients with rheumatoid arthritis, psoriatic arthritis and ankylosing spondylitis: results from the Danish nationwide DANBIO registry. Ann Rheum Dis. 2015;74:e12.

35. Abdelrahman F, Mortada M. Impact of application of ASAS criteria for axial spondyloarthritis on the diagnostic delay in Egyptian patients. Ann Rheum Dis. 2018;77:1556-7 [abstract AB0858].

36. Danve A, Deodhar A. Axial spondyloarthritis in the USA: diagnostic challenges and missed opportunities. Clin Rheumatol. 2019;38:625-34.

37. Taurog JD, Chhabra A, Colbert RA. Ankylosing spondylitis and axial spondyloarthritis. N Engl J Med. 2016;374:2563-74.
38. van den Berg R, Lenczner G, Feydy A, et al. Agreement between clinical practice and trained central reading in reading of sacroiliac joints on plain pelvic radiographs. Results from the DESIR cohort. Arthritis Rheumatol. 2014;66:2403-11.

39. van Tubergen A, Heuft-Dorenbosch L, Schulpen G, et al. Radiographic assessment of sacroiliitis by radiologists and rheumatologists: does training improve quality? Ann Rheum Dis. 2003;62:519-25.

40. Weber U, Jurik AG, Zejden A, et al. Frequency and anatomic distribution of magnetic resonance imaging features in the sacroiliac joints of young athletes: exploring "background noise" toward a data-driven definition of sacroiliitis in early spondyloarthritis. Arthritis Rheumatol. 2018;70: 736-45.

41. Weber U, Lambert RG, Østergaard M, Hodler J, Pedersen SJ, Maksymowych WP. The diagnostic utility of magnetic resonance imaging in spondylarthritis: an international multicenter evaluation of one hundred eighty-seven subjects. Arthritis Rheum. 2010;62(10):3048-58.

42. Lukas C, Cyteval C, Dougados M, Weber U. MRI for diagnosis of axial spondyloarthritis: major advance with critical limitations 'Not everything that glisters is gold (standard)'. RMD Open. 2018;4: $\mathrm{e} 000586$.

43. van der Heijde D, Sieper J, Elewaut D, Deodhar A, Pangan AL, Dorr AP. Referral patterns, diagnosis, and disease management of patients with axial spondyloarthritis: results of an international survey. J Clin Rheumatol. 2014;20:411-7.

44. Magrey M, Yi E, Wolin D, et al. Recognition of inflammatory back pain by US healthcare providers and barriers to specialist referral. Arthritis Rheumatol. 2019;71(Suppl 10) [abstract 631].

45. Magrey M, Yi E, Wolin D, et al. Delayed diagnosis of ankylosing spondylitis: results from a survey of 1690 US physicians from 10 specialties. Ann Rheum Dis. 2019;78(Suppl 2):1247-9.

46. Seo MR, Baek HL, Yoon HH, et al. Delayed diagnosis is linked to worse outcomes and unfavourable treatment responses in patients with axial spondyloarthritis. Clin Rheumatol. 2015;34:1397-405.

47. Hutton B, Salanti G, Caldwell DM, et al. The PRISMA extension statement for reporting of systematic reviews incorporating network meta-analyses of health care interventions: checklist and explanations. Ann Intern Med. 2015;162:777-84.

48. Downs SH, Black N. The feasibility of creating a checklist for the assessment of the methodological quality both of randomised and non-randomised 
studies of health care interventions. J Epidemiol Community Health. 1998;52:377-84.

49. Nie A, Wang C, Song Y, Xie X, Yang H, Chen H. Prevalence and factors associated with disturbed sleep in outpatients with ankylosing spondylitis. Clin Rheumatol. 2018;37:2161-8.

50. Zhao J, Zheng W, Zhang C, Li J, Liu D, Xu W. Radiographic hip involvement in ankylosing spondylitis: factors associated with severe hip diseases. J Rheumatol. 2015;42:106-10.

51. Aggarwal R, Malaviya AN. Diagnosis delay in patients with ankylosing spondylitis: factors and outcomes-an Indian perspective. Clinical Rheumatol. 2009;28:327-31.

52. Gunasekera W, Shaddick A, Jobling A, Smith R, Sengupta R. Diagnostic delay worsens mobility and work disability in ankylosing spondylitis. Ann Rheum Dis. 2014;73:1046 [abstract AB0735].

53. Martindale J, Goodacre L. The journey to diagnosis in AS/axial SpA: the impact of delay. Musculoskelet Care. 2014;12:221-31.

54. Fitzgerald G, Gallagher P, O'Sullivan C, et al. Delayed diagnosis of axial spondyloarthropathy is associated with a higher prevalence of depression. Rheumatology. 2017;56(Suppl 2) [abstract 112].

55. Sullivan C, Quinn K, Harney S, Ryan JG. The use of anti-TNF therapy for ankylosing spondylitis in everyday rheumatology practice and the relationship to disease activity, work disability and diagnostic delay. Ir J Med Sci. 2014;183:579-84.

56. Sullivan C, FitzGerald O. Time to diagnosis of ankylosing spondylitis and the effect on work disability. Ir J Med Sci. 2011;180:S183.

57. Mennini FS, Viti R, Marcellusi A, Sciattella P, Viapiana O, Rossini M. Economic evaluation of spondyloarthritis: economic impact of diagnostic delay in Italy. Clinicoecon Outcomes Res. 2018;10: 45-51.

58. Abdul-Sattar A, Abou El Magd S. Prevalence and associated risk factors for work disability in Egyptian patients with ankylosing spondylitis from one center. Int J Rheum Dis. 2017;20:2028-34.

59. Ibn Yacoub Y, Amine B, Laatiris A, Bensabbah R, Hajjaj-Hassouni N. Relationship between diagnosis delay and disease features in Moroccan patients with ankylosing spondylitis. Rheumatol Int. 2012;32:357-60.

60. Hajialilo M, Ghorbanihaghjo A, Khabbazi A, Kolahi S, Rashtchizadeh N. Ankylosing spondylitis in Iran; late diagnosis and its causes. Iran Red Crescent Med J. 2014;16:e11798.

61. Alayli G, Hartavi A, Bilgici A, Durmus D, Kuru O. Does delay diagnosis in ankylosing spondylitis affect clinical parameters and radiologic progression? Osteoporos Int. 2015;26(Suppl 1) [abstract P670].

62. Cakar E, Taskaynatan MA, Dincer U, Kiralp MZ, Durmus O, Ozgul A. Work disability in ankylosing spondylitis: differences among working and workdisabled patients. Clin Rheumatol. 2009;28: 1309-14.

63. Dincer U, Cakar E, Kiralp MZ, Dursun H. Diagnosis delay in patients with ankylosing spondylitis: possible reasons and proposals for new diagnostic criteria. Clin Rheumatol. 2008;27:457-62.

64. Slobodin G, Reyhan I, Avshovich N, et al. Recently diagnosed axial spondyloarthritis: gender differences and factors related to delay in diagnosis. Clin Rheumatol. 2011;30:1075-80.

65. Cayetti LA, Schneeberger E, Zamora N, Sommerfleck F, Gitera G. How the delay in diagnosis impacts on the clinical, functional and radiographic status of patients with ankylosing spondylitis. Is there a window of opportunity? Arthritis Rheumatol. 2013;64:S657 [abstract 1547].

66. Grigg SE, Martin BJ, Buchanan RR, Schachna L. Burden of delay to diagnosis of ankylosing spondylitis. Arthritis Rheumatol. 2011;63 [abstract 1308].

67. Gran JT, Ostensen M, Husby G. A clinical comparison between males and females with ankylosing spondylitis. J Rheumatol. 1985;12:126-9.

68. Kennedy LG, Will R, Calin A. Sex ratio in the spondyloarthropathies and its relationship to phenotypic expression, mode of inheritance and age at onset. J Rheumatol. 1993;20:1900-4.

69. Deodhar A, Strand V, Kay J, Braun J. The term 'nonradiographic axial spondyloarthritis' is much more important to classify than to diagnose patients with axial spondyloarthritis. Ann Rheum Dis. 2016;75: 791-4.

70. Lee W, Reveille JD, Davis JC Jr, Learch TJ, Ward $\mathrm{MM}$, Weisman $\mathrm{MH}$. Are there gender differences in severity of ankylosing spondylitis? Results from the PSOAS cohort. Ann Rheum Dis. 2007;66:633-8.

71. Tournadre A, Pereira B, Lhoste A, et al. Differences between women and men with recent-onset axial spondyloarthritis: results from a prospective multicenter French cohort. Arthritis Care Res (Hoboken). 2013;65:1482-9. 
72. de Carvalho HM, Bortoluzzo AB, Gonçalves CR, et al. Gender characterization in a large series of Brazilian patients with spondyloarthritis. Clin Rheumatol. 2012;31:687-95.

73. Ibn Yacoub Y, Amine B, Laatiris A, Hajjaj-Hassouni $\mathrm{N}$. Gender and disease features in Moroccan patients with ankylosing spondylitis. Clin Rheumatol. 2012;31:293-7.

74. Landi M, Maldonado-Ficco $\mathrm{H}$, Perez-Alamino $\mathrm{R}$, et al. Gender differences among patients with primary ankylosing spondylitis and spondylitis associated with psoriasis and inflammatory bowel disease in an Iberoamerican spondyloarthritis cohort. Medicine (Baltimore). 2016;95:e5652.

75. Shahlaee A, Mahmoudi M, Nicknam MH, Farhadi E, Fallahi S, Jamshidi AR. Gender differences in Iranian patients with ankylosing spondylitis. Clin Rheumatol. 2015;34:285-93.

76. Stolwijk C, van Tubergen A, Castillo-Ortiz JD, Boonen A. Prevalence of extra-articular manifestations in patients with ankylosing spondylitis: a systematic review and meta-analysis. Ann Rheum Dis. $2015 ; 74: 65-73$.

77. Zarco P, González CM, Rodríguez de la Serna A, et al. Extra-articular disease in patients with spondyloarthritis. Baseline characteristics of the spondyloarthritis cohort of the AQUILES study. Reumatol Clin. 2015;11:83-9.

78. Mitulescu TC, Popescu C, Naie A, et al. Acute anterior uveitis and other extra-articular manifestations of spondyloarthritis. J Med Life. 2015;8: 319-25.

79. Sieper J, van der Heijde D. Review: nonradiographic axial spondyloarthritis: new definition of an old disease? Arthritis Rheum. 2013;65:543-51.

80. Rusman T, van Vollenhoven RF, van der HorstBruinsma IE. Gender differences in axial spondyloarthritis: women are not so lucky. Curr Rheumatol Rep. 2018;20:35.

81. Kiltz U, Baraliakos X, Karakostas P, et al. Do patients with non-radiographic axial spondylarthritis differ from patients with ankylosing spondylitis? Arthritis Care Res (Hoboken). 2012;64:1415-22.

82. Salaffi F, Carotti M, Garofalo G, Giuseppetti GM, Grassi W. Radiological scoring methods for ankylosing spondylitis: a comparison between the Bath Ankylosing Spondylitis Radiology Index and the modified Stoke Ankylosing Spondylitis Spine Score. Clin Exp Rheumatol. 2007;25:67-74.
83. Başkan BM, Sivas F, Inal EE, et al. Comparison of the Bath Ankylosing Spondylitis Radiology Index and the modified Stoke Ankylosing Spondylitis Spine Score in Turkish patients with ankylosing spondylitis. Clin Rheumatol. 2010;29:65-70.

84. Fernández-Carballido C, Navarro-Compán V, Castillo-Gallego C, et al. Disease activity as a major determinant of quality of life and physical function in patients with early axial spondyloarthritis. Arthritis Care Res (Hoboken). 2017;69:150-5.

85. Machado P, Landewé R, Braun J, Hermann KG, Baker D, van der Heijde D. Both structural damage and inflammation of the spine contribute to impairment of spinal mobility in patients with ankylosing spondylitis. Ann Rheum Dis. 2010;69: 1465-70.

86. Calin A, Elswood J, Rigg S, Skevington SM. Ankylosing spondylitis-an analytical review of 1500 patients: the changing pattern of disease. J Rheumatol. 1988;15:1234-8.

87. Jovaní V, Blasco-Blasco M, Ruiz-Cantero MT, Pascual E. Understanding how the diagnostic delay of spondyloarthritis differs between women and men: a systematic review and metaanalysis. J Rheumatol. 2017;44:174-83.

88. van der Horst-Bruinsma IE, Zack DJ, Szumski A, Koenig AS. Female patients with ankylosing spondylitis: analysis of the impact of gender across treatment studies. Ann Rheum Dis. 2013;72: 1221-4.

89. Webers C, Essers I, Ramiro S, et al. Gender-attributable differences in outcome of ankylosing spondylitis: long-term results from the Outcome in Ankylosing Spondylitis international study. Rheumatology (Oxford). 2016;55:419-28.

90. Glintborg B, Ostergaard M, Krogh NS, Dreyer L, Kristensen HL, Hetland ML. Predictors of treatment response and drug continuation in 842 patients with ankylosing spondylitis treated with anti-tumour necrosis factor: results from 8 years' surveillance in the Danish nationwide DANBIO registry. Ann Rheum Dis. 2010;69:2002-8.

91. Kristensen LE, Karlsson JA, Englund M, Petersson IF, Saxne T, Geborek P. Presence of peripheral arthritis and male sex predicting continuation of anti-tumor necrosis factor therapy in ankylosing spondylitis: an observational prospective cohort study from the South Swedish Arthritis Treatment Group Register. Arthritis Care Res (Hoboken). 2010;62:1362-9.

92. Ogdie A, Nowell WB, Reynolds R, et al. Diagnosis journey of patients with ankylosing spondylitis in the United States. Ann Rheum Dis. 2018;77:631-2. 